\title{
Comparison of Routing Algorithms using Riverbed Modeler
}

\author{
Suraj Wandile ${ }^{1}$, Rohit Zende ${ }^{2}$, Siddhant Sribhashyam ${ }^{3}$, Mrs. M. R. Kale ${ }^{4}$, Mr. R. C. Jaiswal ${ }^{5}$ \\ Student, E\&TC Department, Pune Institute of Computer Technology, Pune, India ${ }^{1,2,3}$ \\ Guide, Assistant Professor, E\&TC Department, Pune Institute of Computer Technology, Pune, India ${ }^{4}$ \\ Mentor, Associate Professor, E\&TC Department, Pune Institute of Computer Technology, Pune, India ${ }^{5}$
}

\begin{abstract}
One of the vital practice in the internet is routing of data packets. A routing protocol specifies the method of communication among routers used in the inter connection of networks. A routing table in the memory of a router keeps track of routes to particular network destination. There are varios routing protocols which have application on the internet like Routing Information Protocol (RIPv2), Open Shortest Path First (OSPF), Interior Gateway Routing Protocol (IGRP), Enhanced Interior Gateway Routing Protocol (EIGRP), Intermediate System To Intermediate System (IS-IS), etc. Every single protocol has its unique way of packet routing. This paper presents a simple relative study of RIP, OSPF, EIGRP and IS-IS dynamic routing protocols. The Routing Information Protocol (RIP) comes under the distance vector algorithm while Enhanced Interior Gateway Routing Protocol (EIGRP) is an advanced distance-vector routing protocol, the Open Shortest Path First Protocol (OSPF) is a link state routing algorithm. Intermediate System to Intermediate System (IS-IS) regulates the superlative route for information through a packet-switched network. The crucial objective of this research work is to illustrate the comparitive performance analysis of dynamic routing protocols and redistribution among the protocols. Cisco routers were used in our simulated network topology.
\end{abstract}

Keywords: Routing Protocols, Topology, Convergence, Comparison.

\section{INTRODUCTION}

In the last decade, thanks to the increasing innovation of telecommunications technology, we have seen the surge in number of internet users in developing countries. It is possible to exchange the routing information between routers through the routing protocols. Routing protocols based on certain algorithms permits routers to inform about remote networks dynamically and add this information to their routing tables automatically.

To identify the optimum path to each network, routing protocols are used and added to the routing table. The fundamental advantage of using dynamic routing protocol is that whenever there is topology change routers exchange routing information, which permits routers to learn certainly not only about alien networks but also to find substitute paths if there is a link failure to a running network.

In comparison with static routing, less administrative overhead is required in dynamic routing protocols. Routing protocols let routers to share data about remote networks dynamically and add this information to their routing tables spontaneously. Besides, to meet the demands of changing network requirements dynamic routing protocols have evolved over several years. Individual router comprises particulars of its neighboring networks only. A routing protocol shares this information first among immediate neighbors, and then throughout the network.

This way, routers gain knowledge of the topology of the network. Several organizations have shifted towards more recent routing protocols, for example Enhanced Interior Gateway Routing Protocol (EIGRP) and Intermediate systems

To Intermediate systems (IS-IS).Figure-I shows how dynamic routing protocols are classified.

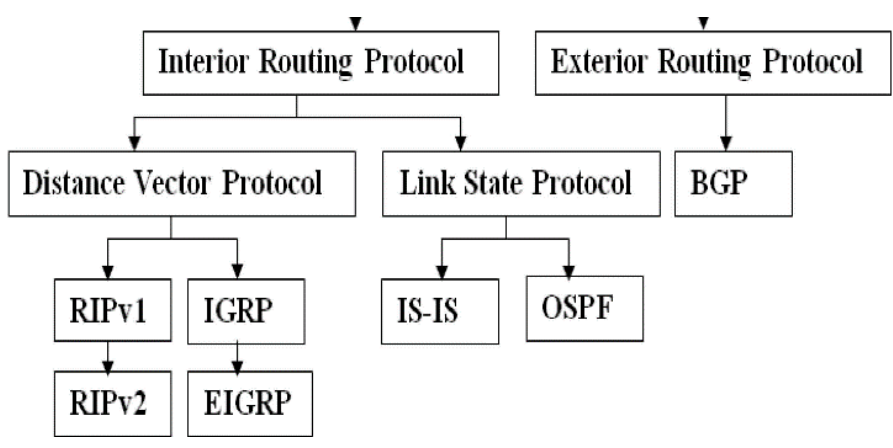


Vol. 6, Issue 6, June 2017

\section{PERFORMANCE ANALYSIS}

Riverbed Modeler is used as simulation tool for Performance Analysis

\section{A. Metrics}

The best path is decided by the Routing protocols. These routing protocols make use of metrics for decision. Several factors plays vital role in dynamic routing protocols to decide the ideal or shortest pathway to exact target. For example RIP uses hop count (number of hops) to determine the best possible route, OSPF make use of bandwidth as metric, and EIGRP uses a mixture of delay and bandwidth as metric.

\section{(1) RIPv2 - Hop Count}

RIPv2 uses hop count as a metric value. The hop count Corresponds to the number of routers a packet must cross to reach the destination node. The RIPv2 chose the route with the lowest hop count.

(2) OSPF - cost

Open Shortest Path First (OSPF) uses "Cost" as a metric and practices a Reference Bandwidth of $100 \mathrm{Mbps}$ for cost calculation. The formula to estimate the metric is Reference Bandwidth divided by Interface Bandwidth.

\section{(3) EIGRP - Delay and Bandwidth}

EIGRP uses various values in its composite metric to calculate the best path in a network. Delay, Bandwidth, Load and Reliability. Best path is selected with the smallest composite metric value calculated from these multiple parameters. In general, delay and bandwidth as default parameters.

\section{(4) IS-IS - Narrow and Wide}

There are two basic metric types Narrow and Wide and another one state is the transitional state. The transitional mode is used when migrating from narrow to wide.

\section{B. Convergence}

Convergence is achieved when all the routers of a network have the same topological data. When all the routers throughout the network have consistent data in their respective routing table. The network achieves converge when all routers have comprehensive and precise data about the network under consideration. Convergence is a vital concept for a set of routers that engage in dynamic routing. Every Interior Gateway Routing Protocol depend on convergence for optimum operation. Convergence time is the time necessary by the routers to update the routing table, calculate the best path and share the data throughout the network. BGP, an exterior gateway routing protocol never usually convergence in a network as big as the Internet. The network's routing protocol must converge for reliable operation.

\section{Throughput}

The units of information a system can process in a given amount of time is throughout. Traffic throughput of a network is delimited by the routing protocol in consideration, and the router hardware, a crucial theme for many network administrators. Throughput is useful to systems from several parts of CPU and network systems to organizations. Associated procedures of system efficiency include the time for some certain workload to be completed, acknowledgment of response time, the amount of time between a single interactive user request.

\section{TABLE I}

\begin{tabular}{|c|c|c|c|c|}
\hline Feature & RIP & OSPF & EIGRP & IS-IS \\
\hline Type & Distance Vector & Link state & Hybrid & Link state \\
\hline Algorithm & Bellman-ford & Dijkstra & DUAL & Dijkstra \\
\hline Class full/class less & $\begin{array}{l}\text { V1 : class full } \\
\text { V2 : class less }\end{array}$ & Class less & Class less & Class less \\
\hline Metric & Hop count & Cost & Bandwidth/Delay & Narrow and Wide \\
\hline $\begin{array}{l}\text { Timers update } \\
\text { (Hello/Dead ) }\end{array}$ & $30 \mathrm{sec}$ & $\begin{array}{l}\text { Triggered when } \\
\text { network change } \\
\text { occurs, send periodic } \\
\text { update LSA refreshes } \\
\text { every } 30 \text { minutes }\end{array}$ & $\begin{array}{l}\text { Triggered } \quad \text { (LAN } \\
5 / 15, \text { WAN 60/180) }\end{array}$ & \\
\hline $\begin{array}{l}\text { Administrative } \\
\text { distance }\end{array}$ & 120 & 110 & $\begin{array}{l}\text { Internal } 90 \\
\text { External } 170\end{array}$ & 115 \\
\hline Authentication & V1: No & MD5 Authentication & MD5 Authentication & MD5 Authentication \\
\hline
\end{tabular}


Vol. 6, Issue 6, June 2017

\begin{tabular}{|l|l|l|l|l|}
\hline & V2 $:$ Yes & & & \\
\hline Hop limit & 15 & 235 & None & \\
\hline Convergence & Slow & Fast & Very fast & Fast \\
\hline Type of updates & Full table & Only changes & Only changes & \\
\hline Support VLSM & $\begin{array}{l}\text { V1 : No } \\
\text { V2 }: \text { Yes }\end{array}$ & Yes & Yes & Yes \\
\hline Network size & Small & Large & Large & Large \\
\hline $\begin{array}{l}\text { Split Horizon } \\
\text { sensitive }\end{array}$ & No & Yes & Yes & Yes \\
\hline Area Types & - & $\begin{array}{l}\text { Backbone, stubby, } \\
\text { Not so-stubby, totally } \\
\text { stubbing }\end{array}$ & - & - \\
\hline Latency & Less & Less & Less \\
\hline
\end{tabular}

Primary differences of RIP, OSPF, EIGRP, IS-IS protocols response time, the amount of time between a single interactive user request and receipt of the response.

\section{RESULTS AND ANALYSIS}

A. Network Convergence Activity



The first pseak represent initialization. The next peak after initialization is failure and after that is recover peak and so on. The convergence time depends on the width of peak. If the peak is wider, the protocols converges slowly. In this mesh topology, IS-IS is the fastest protocol among all four. EIGRP is slight faster than RIP. It is clear from the graph that OSPF is slowest.

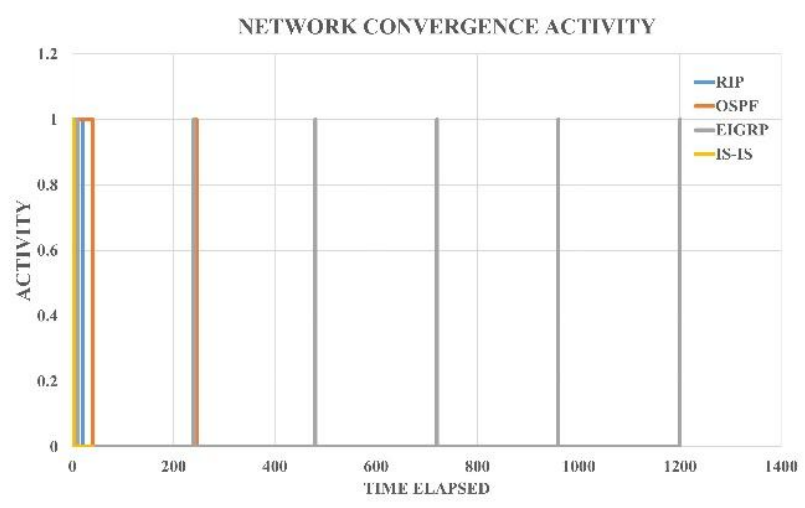

Convergence activity in a 50 nodes tree network for different routing protocols. IS-IS is the fastest among all four. OSPF has a longer initialization time compare to RIP, EIGRP and IS-IS. Both OSPF and RIP has longer initialization time as compared to EIGRP and IS-IS. EIGRP has longer initialization time than IS-IS. 
B. Network Convergence Duration

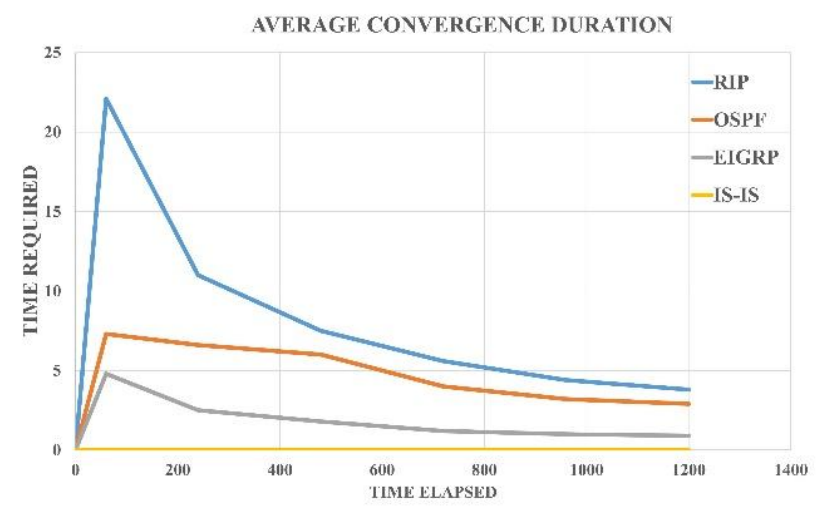

Convergence duration for 50-node tree network for different protocols. IS-IS is still the fastest. Time required for EIGRP convergence is more than IS-IS. OSPF take a slight longer time than EIGRP and it is clear from the graph that RIP is the slowest and take a considerably lot more time than OSPF.

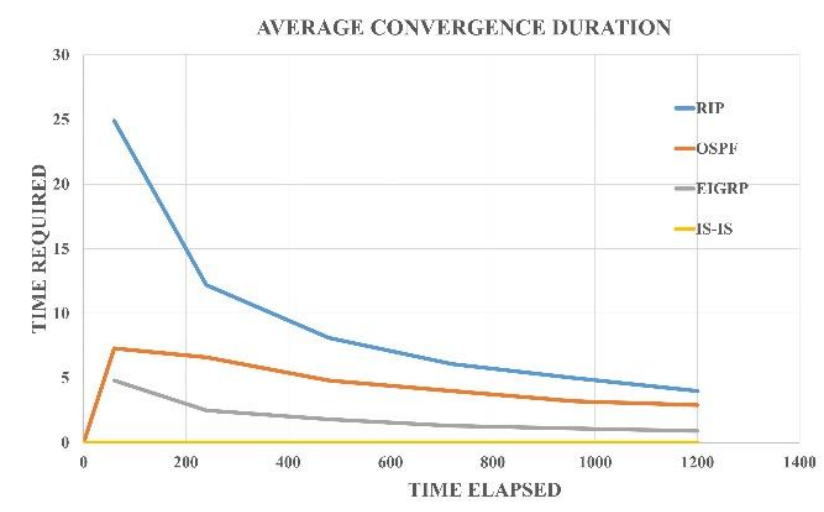

As concluded from the graph, IS-IS is still faster is every aspect i.e. faster initialization time and faster convergence duration. OSPF is the second fastest in convergence but has some delay in initialization. EIGRP has zero initialization time but converges after OSPF. RIP is the slowest in convergence and it also has some delay in initialization.

C. Traffic Sent

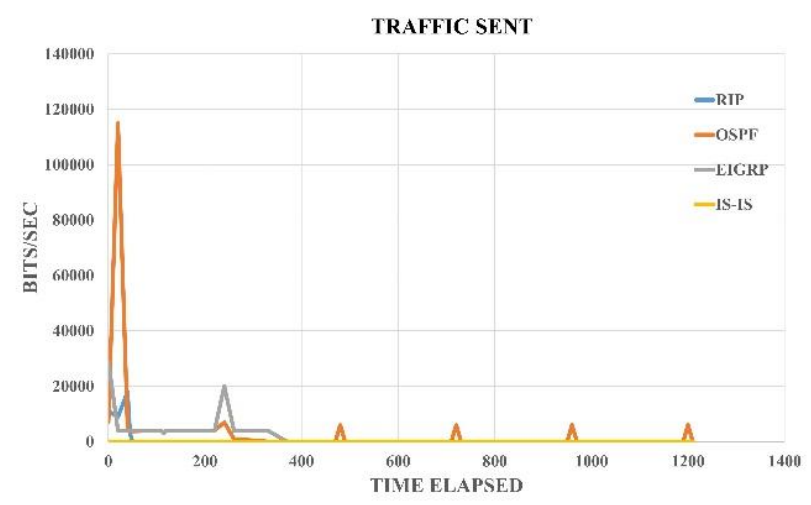

The figure above shows the router traffic sent in bits/sec in four protocols using mesh topology. The first peak in this figure represents initial traffic, the next peak is link failure and the peak after that is link recovery. The figure clearly signifies that OSPF has the highest initial traffic since OSPF will map whole network, which requires routers to distribute a large amount of information. Also, we observe that IS-IS has the highest bandwidth efficiency, and second one is EIGRP. RIP update its routing table every 30 seconds hence RIP shows a little difference than OSPF and EIGRP. 


\section{CONCLUSION}

We studied the performance of four major types of routing protocols: RIP, OSPF, EIGRP and IS-IS using Riverbed Modeler. Tree and Mesh topologies had been built and the simulation of each routing protocols in all three topologies had been performed. At first implemented the three routing protocols into a small tree network and evaluated the convergence activity, convergence duration and traffic sent (bytes/sec) to compare the difference in their performance and after that, we implemented the three protocols into large mesh and large tree topologies and evaluated the same three parameters. IS-IS is the fastest among all four. OSPF has a longer initialization time compare to RIP, EIGRP and IS-IS. Both OSPF and RIP has longer initialization time as compared to EIGRP and IS-IS. EIGRP has longer initialization time than IS-IS.

\section{ACKNOWLEDGMENT}

This project work was partially supported by Project Coordinator Mr. R C Jaiswal we thank him for his help. We thank our Project Guide Mrs. M R Kale, Professor, Pune institute of computer technology, Pune, who provided insight and expertise that greatly assisted the project work.

\section{REFERENCES}

[1] Jagdeep Singh, Rajiv Mahajan, "Simulation Based comparitive study of RIP, OSPF and EIGRP”, International Journal of Advanced Research in Computer Science and Software Engineering, Vol3, Iss.8, pp.1-4, August 2013.

[2] P. Rakheja, P. Kaur, A. Gupta and A. Sharma, "Performance Analysis of RIP, OSPF, IGRP and EIGRP Routing Protocols in a Network" International Journal of Computer Applications (0975 - 888) Volume 48- No.18, June 2012.

[3] Vetriselvan.V, Mahendran.M , "Survey on RIP, OSPF and EIGRP Routing protocols", International Journal of Computer Science and Information Technology, Vol.5(2), iss.3, pp.2-7, 2014.

[4] Pankaj Rakheja, Prabhjot Kaur, Anjali Gupta, Aditi Sharma, "Performance Analysis of RIP, OSPF,IGRP and EIGRP Routing Protocols in a IEEE Sponsored 2nd International Conference on Innovations in Information Embedded and Communication Systems ICIIECS'15 Network", International Journal of Computer Applications(0975-888)Volume 48-No.18, pp.210-224,2012.

[5] Song Yang, Zhang Zhi Yong, "RIP Internet Protocol Failure Analysis and Research", Proceedings of 2012 International Conference on Industrial Control and Electronics Engineering.

[6] Jui-Fa Chen, Wei-Chuan Lin, "A Message Interchange Protocol based on Routing Information Protocol in a Virtual World", Proceedings of 19th International Conference on Advanced Information Networking and Applications (AINA’05).

[7] Meenakshi, Akhil Kaushik, Satvika, "QoS Parameter Evaluation of UMTS under EIGRP, IGRP and RIP using OPNET", International Conference on Communication and Signal Processing, April 3-5, 2014.

[8] Bohdanowicz, H. Dickel, and C. Steigner. Metric-based topology investigation. International Conference on Networking, 0:176-184, 2009.

[9] S. G. Thornier, "Dynamic Routing protocol implementation decision between EIGRP, OSPF and RIP based in technical background using OPNET Modeler", in Proc.Second International Conference on Computer and Network Technology(ICCNT), Bangkok, Thailand, Pp 191195,2010 .

[10] Li Lan, Li Li , Chen Jianya, "A Multipath Routing Algorithm Based on OSPF Routing Protocol" 2012 Eighth International Conference on Semantics, Knowledge and Grids (SKG), pp. 269-272,2012.

[11] Ittiphon krinpayorm , Suwat Pattaramalai," Link Recovery Comparison Between OSPF \& EIGRP"', International Proceedings of Computer Science \& Information Technology, Vol. 27,pp.192-197,2012. 